\title{
Nonmigrating diurnal tides as measured by the TIMED Doppler Interferometer: Preliminary results
}

\author{
J. Oberheide ${ }^{\mathrm{a}}, \mathrm{Q} . \mathrm{Wu}^{\mathrm{b}}, \mathrm{D}$. A. Ortland ${ }^{\mathrm{c}}$, T. L. Killeen ${ }^{\mathrm{b}}$, \\ M. E. Hagan ${ }^{\mathrm{b}}$, R. G. Roble ${ }^{\mathrm{b}}$, R. J. Niciejewski ${ }^{\mathrm{d}}$, and \\ W. R. Skinner ${ }^{\mathrm{d}}$ \\ ${ }^{a}$ Physics Department, University of Wuppertal, Gauss Strasse 20, 42097 \\ Wuppertal, Germany \\ ${ }^{\mathrm{b}}$ High Altitude Observatory, National Center for Atmospheric Research, Boulder, \\ CO, USA \\ ${ }^{\mathrm{c}}$ Northwest Research Associates, Bellevue, Washington, USA \\ ${ }^{\mathrm{d}}$ Space Physics Research Laboratory, Department of Atmospheric, Oceanic, and \\ Space Sciences, The University of Michigan, Ann Arbor, USA
}

\begin{abstract}
Preliminary meridional wind data from the TIMED Doppler Interferometer (TIDI) onboard the TIMED satellite are analyzed for nonmigrating diurnal tides. Tidal definitions are given for the most pronounced westward, eastward and standing oscillations (w2, e3, s0). The analysis interval is March 2002 to June 2004 and covers the altitude range $85-105 \mathrm{~km}$. Monthly tidal wind fields from $40^{\circ} \mathrm{S}-40^{\circ} \mathrm{N}$ are presented. TIDI tides compare favorably with previously reported $95 \mathrm{~km}$ HRDI results. Nonmigrating diurnal tidal wind speeds larger than $30 \mathrm{~m} / \mathrm{s}$ are observed thus emphasizing the important role of nonmigrating tides in middle atmosphere coupling. A comparative analysis with the Global Scale Wave Model (GSWM) and the Thermosphere-Ionosphere-Mesosphere-Electrodynamics General Circulation Model (TIME-GCM) indicates that both latent heat release in the tropical troposphere and non-linear Planetary Wave / migrating tide interaction are important sources of nonmigrating tides in the mesosphere and lower thermosphere.
\end{abstract}

Key words: nonmigrating tides, MLT region, atmospheric coupling, wind, TIDI, TIMED, GSWM, TIME-GCM

Email address: joberh@uni-wuppertal.de (J. Oberheide).

$U R L$ : www.crista.uni-wuppertal.de (J. Oberheide). 


\section{Introduction}

Tidal waves play a key role in coupling the lower with the middle and upper atmosphere by propagating up and away from their tropospheric and stratospheric sources thus transporting energy and momentum to upper altitudes. Tides are global-scale waves with periods that are harmonics of a solar day (e.g. Chapman and Lindzen, 1970). They are among the most striking dynamical features in the mesosphere and lower thermosphere (MLT). Probably, the best known class of tidal oscillations are the Sun-synchronous or migrating tides. Migrating tides propagate westward with the apparent motion of the Sun and are primarily driven by the zonally symmetric absorption of solar IR and UV radiation by tropospheric water and water vapor and stratospheric ozone. The zonal wavenumbers $n$ of the migrating tides are therefore equal to their frequencies (in cycles per day), e.g. $n=1$ for the prominent migrating diurnal (24h period) tide.

The second class of tidal oscillations are the non-Sun-synchronous or nonmigrating tides. Their zonal wavenumbers are not equal to their frequencies (in cycles per day) and they can propagate westward, eastward or remain standing. Nonmigrating tides are the focus of the present work and only tides of diurnal (24 hours) period will be discussed in the following. The nomenclature used here is standard: Tidal waves (also called tidal components) are identified by using a letter/number combination indicating both propagation direction ( $w$ : westward, $s$ : standing, $e$ : eastward) and zonal wavenumber $(n \geq 0)$, i.e. $w 1$ is the migrating diurnal tide (westward, zonal wavenumber 1 ), w2 is the westward propagating nonmigrating diurnal tidal component of zonal wavenumber $2, s 0$ is the standing oscillation (having naturally zonal wavenumber 0 ), and $e 3$ is the eastward propagating diurnal component of zonal wavenumber 3 .

Nonmigrating tides may be forced by a variety of quite different mechanisms. Two major sources are latent heat release in the tropical troposphere (e.g. Hagan and Forbes, 2002) and nonlinear interactions between Quasi-Stationary Planetary Waves (QSPW) and the migrating tide (e.g. Hagan and Roble, 2001). Owing to limitations of both the satellite-borne (local time coverage) and ground-based instruments (spatial coverage), global observations of nonmigrating tides are still quite limited. Nonmigrating tides in equatorial MLT temperatures were measured by the CRISTA instrument. The observed signatures could be understood by a combination of both latent heat and wavewave interaction forcing (Oberheide et al., 2002; Oberheide and Gusev, 2002). Lieberman et al. (2004) studied nonmigrating tides in LIMS temperatures below $80 \mathrm{~km}$ and found evidence for wave-wave interaction forcing in the stratosphere. Forbes et al. (2003), Manson et al. (2002, 2004), and Huang and Reber (2004) were able to retrieve nonmigrating tidal amplitudes and phases from HRDI/UARS wind data. The specifics of the HRDI instrument limited their 
analyses to $95 \mathrm{~km}$ but they clearly showed the month-to-month variability of the nonmigrating tides as well as the resulting longitude modulation of the diurnal tidal amplitude. With a different analysis approach Talaat and Lieberman (1999) retrieved nonmigrating tidal information between 60 and $120 \mathrm{~km}$ from HRDI but without being able to clearly identify the zonal wavenumbers of the tidal components.

This work provides monthly tidal definitions of the diurnal components $w 2, s 0$, and $e 3$ from preliminary wind measurements of the TIMED Doppler Interferometer (TIDI) flying on the TIMED satellite that was launched in December 2001. Only meridional winds are presented. Zonal winds will be discussed in a forthcoming publication. Altitudes covered are 85-105 km and the latitude range is $40^{\circ} \mathrm{S}-40^{\circ} \mathrm{N}$. The TIDI results will be compared to the abovementioned HRDI results at $95 \mathrm{~km}$. Tidal sources will be discussed by comparing the measurements to two sets of model predictions: i) the Global Scale Wave Model (GSWM, version 02) that accounts for latent heat release in the tropical troposphere (Hagan and Forbes, 2002) and ii) to the Thermosphere Ionosphere Mesosphere Electrodynamics General Circulation Model (TIME-GCM) (Roble and Ridley, 1994) that accounts for PW/tidal interaction.

\section{TIDI measurements and data processing}

The Thermosphere Ionosphere Mesosphere Energetics and Dynamics (TIMED) satellite was launched in December 2001. One of the instruments onboard is the TIMED Doppler Interferometer (TIDI) with the primary objective to measure winds in the MLT region.

The TIDI instrument was developed and built by the University of Michigan (Killeen et al., 1999). TIDI measures neutral winds by performing limb scans of various upper atmosphere airglow layers and monitoring the Doppler shift of airglow emissions induced by neutral winds. The four TIDI telescopes are orthogonally oriented, allowing the instrument to measure neutral wind vectors on both sides of the satellite track (i.e., cold and warm sides). The two telescopes viewing the same side of the track observe the same locations with a time delay of a few minutes as the spacecraft moves forward. The viewing directions of these two telescopes are perpendicular to one another. Assuming that the neutral winds do not change much during the short time period, the samplings at the two directions can then be used to form the neutral wind vector in terms of the meridional and zonal components. There are four local time samplings at latitudes equatorwards of $\pm 60^{\circ}$ and two at latitudes poleward of $\pm 60^{\circ}$. The local time coverage pattern shifts slowly (by $0.2 \mathrm{hr} /$ day) towards earlier local times as time progresses. The satellite orbits the Earth 15 times per day (98 minutes per orbit), and the local time of the orbit does 
not change much within a day. As the Earth rotates under the TIMED orbit, however, TIDI provides longitudinal coverage around the globe. The four telescopes scan various airglow emissions in a repeating sequence called a scan cycle, which defines the operational mode. Both the daytime cycle and nighttime cycle last for about 100 seconds. That translates to roughly $6^{\circ}$ latitudinal spatial resolution based on the satellite velocity. The $\mathrm{O}_{2}(0-0)$ band P9 line measurement covers the 70 to $110 \mathrm{~km}$ altitude range during the day and 80 to $105 \mathrm{~km}$ during the night. The inverted wind profiles have an altitude resolution of $2.5 \mathrm{~km}$. The TIDI instrument is affected by a light leak from the Earth's reflection of sunlight. Hence, the signal background was higher than expected resulting in increased wind errors (Skinner et al., 2003). Individual inverted wind profiles above $85 \mathrm{~km}$ altitude have an error of about $30 \mathrm{~m} / \mathrm{s}$ during the day and double that during the night.

The TIDI data used here are P9 vector winds (level 3, preliminary data versions 00_01 (2002), 01_01 to 01_03 (2003), 03_00 (2004)) that were produced by the National Center for Atmospheric Research (NCAR, see http://timed.hao.ucar.edu/tidi).

Tides in the TIDI winds are derived by means of a two-dimensional Fourier transform of a 60-day composite data set. The composite data are analyzed as follows:

(1) Combine 5 days of consecutive TIDI meridional wind measurements. This reduces the noise level and closes data gaps. The corresponding 1 hour local solar time (LST) smoothing does not affect the analysis results. Combining 3 days of data yielded almost identical amplitudes and phases (not shown).

(2) Split the combined data set into four subsets of ascending (asc) and descending (dsc) orbit node measurements for the TIDI warm and cold sides respectively. All four datasets have different local times for a given latitude.

(3) Map the 4 subsets separately onto a horizontal grid of $5^{\circ} \times 5^{\circ}$ using a two-dimensional triangular filter function with a full width of $7.5^{\circ}$ in the NS direction and $45^{\circ}$ in EW direction.

(4) Remove the zonal mean from the 4 mapped subsets. The zonal mean removal accounts for potential zero wind line inconsistencies in the preliminary TIDI data. It also removes the migrating tides from the mapped data because migrating tides are observed as zonally symmetric features. See Oberheide et al. (2003) for details of tidal sampling issues in the data from slowly precessing satellites.

(5) Repeat steps 1 . to 4 . in a running mean sense for a 60 day period. The TIMED orbit geometry is such that 60 days of combined TIDI asc/dsc, warm/cold side data subsets have 24 hours of LST coverage.

(6) Combine 60 days of mapped data subsets and sort them in local time. 
Interpolate the sorted data set onto a fixed LST grid, including averaging if more than one data subset is available for a given LST. The resulting composite data set is therefore evenly spaced in LST (24 hours LST coverage) and longitude (360 ${ }^{\circ}$ coverage) for each latitude and altitude.

(7) Compute wavenumber/frequency pairs with two-dimensional Fourier transform . Owing to the 60-day averaging, the results must be interpreted in a climatological sense. Short-time variations will be smoothed out. Account for the fact, that the observed wavenumber is shifted by 1 as compared to the real zonal wavenumber (e.g. diurnal w2 is observed as w1). This is due to the satellite sampling (Oberheide et al., 2003). Assign the amplitudes and phases to the day in the middle of the 60-day period.

(8) Repeat steps 1 . to 7 . in a running mean sense for the analysis period (March 2002 - June 2004) and average the derived amplitudes and phases into monthly bins.

Monthly amplitudes of diurnal components $s 0, w 2$, and $e 3$ at $95 \mathrm{~km}$ are shown in Figure 1. Propagation of the abovementioned wind errors through the analysis procedure yields an amplitude precision of $1 \mathrm{~m} / \mathrm{s}$ and a phase precision of 1.5 hours (for amplitudes $\geq 1 \mathrm{~m} / \mathrm{s}$ ). These are the precisions before the monthly binning. Skinner et al. (2003) describes the optical performance of the TIDI instrument over the course of the analysis period, i.e. the decrease in throughput due to ice deposition on some parts of the optics and the efforts to sublimate the frost that led to an improved instrument performance since April 2003. However, the effect of those optical performance changes on the derived amplitudes and phases appears to be rather small. Both time-series show no apparent signatures of e.g. de-icing efforts. Nevertheless, it cannot be completely precluded that the 2002 tidal amplitude and phase errors are somewhat larger than those for 2003 and 2004. A closer analysis is beyond the scope of the present work, particularly when considering the preliminary nature of the data set. It will be carried out in the future.

Fourier transform of composite data is inherently subject to aliasing from other wavenumber/frequency components. Applying the same analysis to TIMEGCM model predictions that were mapped to the LST, longitude, and latitude of the TIDI measurements showed that aliasing effects (mainly due to QSPW-1) are limited to latitudes $>40^{\circ}$ (Figure not shown). The following discussion and validation of the TIDI results is therefore limited to latitudes equatorwards of $\pm 40^{\circ}$.

\section{Monthly tidal wind fields and altitude structure}

Observations of nonmigrating tides have so far been very sparse. This is, however, different at $95 \mathrm{~km}$ altitude where climatological amplitudes from HRDI 
on UARS are available. Figure 1 shows a comparison of the TIDI amplitudes for $s 0, w 2$, and $e 3$ with the corresponding amplitudes from HRDI. The latter are taken from Forbes et al. (2003) and based upon the observational period 1991-1994. The HRDI amplitudes were also derived by means of Fourier transform on data averaged over one yaw period. The month-to-month variability in both climatological data sets is almost identical though differences in the absolute values of the amplitudes exist.

The $s 0$ amplitudes in both data sets have maxima at $\pm 20^{\circ}$ with a minimum at the equator. The amplitudes are larger in the SH with a peak in Jun-Aug. The NH maximum in Aug-Oct is also present in both TIDI and HRDI observations. For the $w 2$ case, both TIDI and HRDI show amplitude maxima at $\pm 20^{\circ}$ with peaks in February, Oct-Nov and a smaller one in Jun-Jul.

The $s 0$ and $w 2$ amplitudes from TIDI are about 30\%-50\% larger than the HRDI amplitudes in Figure 1. Recent results (Huang and Reber, 2004) of nonmigrating tides in HRDI winds also indicated larger (30\%-50\%) amplitudes than those reported by Forbes et al. (2003). In contrast, the analysis of Manson et al. (2002) of the same data set is more consistent with the Forbes et al. (2003) results. These inconsistencies have yet to be resolved, but it has been speculated (Huang and Reber, 2004) that the specifics of the horizontal data binning might be an issue. A forthcoming analysis of smoothing effects due to horizontal mapping by means of TIDI sampled TIME-GCM results can hopefully contribute to answer this question. It also has yet to be determined whether the small $e 3$ amplitudes from TIDI (as compared to HRDI) are associated with the horizontal binning or whether this difference is more due to comparing data sets from different years.

In order to give an overview of the general structure of the nonmigrating tides seen by TIDI, Figure 2 shows the annual mean amplitudes of components $s 0$, $w 2$, and $e 3$ as function of latitude and altitude. Figure 2 also provides the corresponding phases, but not as annual mean because the variety of tidal sources and the interaction with the changing background atmosphere shifts the phases over the course of a year. The plot therefore gives the phases for the month with pronounced amplitudes for the respective tidal component. Components $s 0$ and $w 2$ show maxima at about $20^{\circ} \mathrm{S}$ and $20^{\circ} \mathrm{N}$ with 12 hours phase jumps occurring at the equator such that these components are antisymmetric. In both cases the maximum amplitude is about $10 \mathrm{~m} / \mathrm{s}$ with a peak altitude of $95 \mathrm{~km}$. A slight enhancement in the southern hemisphere ( $\mathrm{SH}$ ) is observed. In contrast, $e 3$ is symmetric to the equator with a maximum amplitude of about $2 \mathrm{~m} / \mathrm{s}$. Owing to the amplitude confinement to the equator and the smooth phase, this tidal component is often called a diurnal Kelvin wave. 


\section{Discussion}

Figure 3 shows a comparison between the TIDI observations of $s 0$ and the predictions of GSWM and TIME-GCM. GSWM accounts for the latent heat source in the tropical troposphere (Hagan and Forbes, 2002) but not for nonlinear wave-wave interactions. Migrating tide/QSPW interaction is the dominant source of nonmigrating tides in TIME-GCM (Hagan and Roble, 2001) that, on the other hand, does not include the latent heat source. The GSWM results are the synthesis of the $s 0$ monthly calculations at $95 \mathrm{~km}$. In contrast, the TIME-GCM results were obtained by extracting the model winds along the TIDI orbit trajectories and processing them in the manner outlined in Section 2. It is quite obvious that no model alone describes the month-to-month variability observed by TIDI. However, both models together can explain most of the observed features. GSWM amplitudes are symmetric to the equator and peak around equinox. The TIDI amplitudes at equinox are generally similar (about $8-10 \mathrm{~m} / \mathrm{s}$ ) but larger in the $\mathrm{SH}$ during fall $(14-16 \mathrm{~m} / \mathrm{s})$. TIME-GCM, however, shows a distinct $\mathrm{SH}$ amplitude maximum between Jul-Oct $(8 \mathrm{~m} / \mathrm{s})$ that is consistent with the SH TIDI peak in July (but shifted by one month) and the observed large SH amplitudes during fall equinox. TIME-GCM also shows a large response $(6 \mathrm{~m} / \mathrm{s})$ in both hemispheres in Jan-Mar that is also observed with similar amplitudes $(8 \mathrm{~m} / \mathrm{s})$ by TIDI. The structure and amplitude of the observed diurnal $s 0$ component is therefore consistent with a combination of latent heat and wave-wave interaction forcing. The latter source appears to be more important for the enhanced tidal activity during solstice and the generally larger SH amplitudes while the latent heat source leads the $s 0$ component during equinox.

A similar result is obtained when comparing the observed $w 2$ components with the models (not shown). The observed secondary peaks in Jun-Jul (Figure 1) are associated with QSPW/migrating tide interaction only while both latent heat and non-linear interaction are equally important sources during the remainder of the year. The structure (location and month of maxima) of the $e 3$ component (Figure 1) is in very good agreement with the GSWM predictions (not shown) though the observed amplitudes are about 50\% smaller than the model predictions. TIME-GCM $e 3$ response is almost zero.

Tidal components not yet discussed ( $w 6$ to $w 3, e 1, e 2, e 4$ to $e 6$ ) show amplitudes $\leq 4 \mathrm{~m} / \mathrm{s}$ with the $e 1$ component being the largest. The importance of nonmigrating tides for the tidal wind field in the MLT region can be seen from Figure 4. It shows the longitude dependence of the tidal amplitudes when superposing the measured $w 6$ to $e 6$ components (with respect to their relative phasing). A wavenumber 2 like structure is observed with a maximum amplitude larger than $30 \mathrm{~m} / \mathrm{s}$. Although not presented here, such an amplitude is on the order of the annual mean amplitude $(25 \mathrm{~m} / \mathrm{s}$ at $95 \mathrm{~km})$ of the migrating 
diurnal tide (Forbes et al., 2003).

\section{Conclusions}

TIDI meridional wind measurements, though still preliminary, provide for the first time a climatology of nonmigrating diurnal tides in the altitude range $85-105 \mathrm{~km}$. The month-to-month variability is in excellent agreement with the $95 \mathrm{~km}$ HRDI results reported by Forbes et al. (2003) though quantitative amplitude differences between TIDI and HRDI still exist. TIDI annual mean amplitudes of components $s 0$ and $w 2$ are about 30-50\% larger than the HRDI values and $30 \%$ smaller for the $e 3$ component. Parts of these differences might be attributable to the specifics of the data binning. The observed altitude of maximum amplitude is $95 \mathrm{~km}$. The latitude/height distribution of the measured phases is consistent with the predictions of the classical tidal theory, such that $w 2$ and $s 0$ are antisymmetric about the equator while $e 3$ is Kelvin wave like, i.e. symmetric about the equator.

By comparing TIDI with GSWM and TIME-GCM model calculations, the observed month-to-month variability could be attributed to a combination of tides forced by latent heat release in the tropical troposphere and non-linear interaction between QSPW-1 and the migrating tide. For $w 2$ and $s 0$ both sources are of comparable importance while $e 3$ appears to be excited by latent heat release only. The June/July maxima in $s 0$ and $w 2$ are purely attributable

to wave-wave interaction forcing. TIDI therefore gives observational evidence that large-scale tropospheric systems that do not propagate into the MLT can nevertheless influence the dynamics (and chemistry) of that region via their imprint upon the tidal fields. The combined nonmigrating response is in the order of the radiatively forced migrating tide and introduces a considerable longitude modulation of the tidal wind field.

\section{Acknowledgments}

We thank Stan Solomon for comments on an initial draft of the manuscript. The National Center for Atmospheric Research is sponsored by the NSF. This work was also partially supported by the NASA TIMED and NSF CEDAR programs, and BMBF grant 50 QV 9802-4. 


\section{References}

Chapman, S., and R. S. Lindzen, Atmospheric Tides, D. Reidel, Norwell, Mass., 1970.

Forbes, J. M., X. Zhang, E. R. Talaat, and W. Ward, Nonmigrating diurnal tides in the thermosphere, J. Geophys. Res., 108(A1), 1033, 2003.

Hagan, M. E., and R. G. Roble, Modeling the diurnal tidal variability with the NCAR TIME-GCM, J. Geophys. Res., 106, 24,869-24,882, 2001.

Hagan, M. E., and J. M. Forbes, Migrating and nonmigrating diurnal tides in the middle and upper atmosphere excited by tropospheric latent heat release, J. Geophys. Res., 107(D24), 4754, 2002.

Huang, F. T., and C. A. Reber, Nonmigrating semidiurnal and diurnal tides at $95 \mathrm{~km}$ based on wind measurements from the High Resolution Doppler Imager on UARS, J. Geophys. Res.,109(D10110), 2004.

Killeen, T. L., W. R. Skinner, R. M. Johnson, C. J. Edmonson, Q. Wu, R. J. Riciejewski, H. J. Grassl, D. A. Gell, P. E. Hansen, J. D. Harvey, and J. F. Kafkalidis, TIMED Doppler Interferometer (TIDI), Proc. SPIE, 3756, 289-301, 1999.

Lieberman, R. S., J. Oberheide, M. E. Hagan, E. E. Remsberg, and L. L. Gordley, Variability of diurnal tides and planetary waves during November 1978 - May 1979, J. Atmos. Sol. Terr. Phys., 66, 517-528, 2004.

Manson, A. H., Y. Luo, and C. Meek, Global distributions of diurnal and semi-diurnal tides: Observations from HRDI-UARS of the MLT region, Ann. Geophys., 20(11), 1877-1890, 2002.

Manson, A. H., C. Meek, M. Hagan, X. Zhang, and Y. Luo, Global distributions of diurnal and semidiurnal tides: Observations from HRDI-UARS of the MLT region and comparison with GSWM-02 (migrating, nonmigrating components), Ann. Geophys., 22(5), 1529-1548, 2004.

Oberheide, J., and O. A. Gusev, Observation of migrating and nonmigrating diurnal tides in the equatorial lower thermosphere, Geophys. Res. Lett., 29(24), 2167, 2002.

Oberheide, J., M. E. Hagan, R. G. Roble, and D. Offermann, Sources of nonmigrating tides in the tropical middle atmosphere, J. Geophys. Res., 107(D21), 4567, 2002.

Oberheide, J., M. E. Hagan, and R. G. Roble, Tidal signatures and aliasing in temperature data from slowly precessing satellites, J. Geophys. Res., 108(A2), 1055, 2003.

Roble, R. G., and E. C. Ridley, A thermosphere-ionosphere-mesosphereelectrodynamics general circulation model (TIME-GCM): Equinox solar cycle minimum simulations (30-500 km), Geophys. Res. Lett., 21, 417-420, 1994.

Skinner, W. R., and 11 co-authors, Operational Performance of the TIMED Doppler Interferometer (TIDI), Proc. SPIE, 47-57, 2003.

Talaat, E. R., and R. S. Lieberman, Nonmigrating diurnal tides in mesospheric and lower thermospheric winds and temperatures, J. Atmos. Sci., 56(24), 
4073-4087, 1999. 

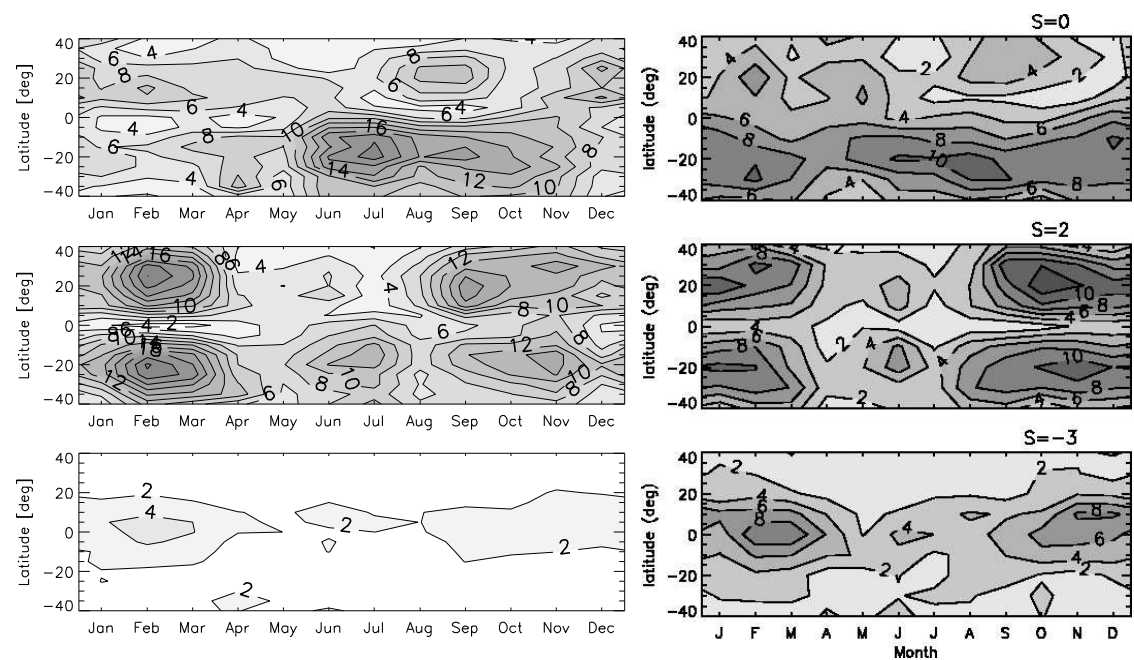

Fig. 1. Diurnal tidal amplitudes $(\mathrm{m} / \mathrm{s})$ for the meridional wind at $95 \mathrm{~km}$. Left panel: TIDI; right panel HRDI. From top to bottom: $s 0, w 2, e 3$. HRDI data are taken from Figure 4 of Forbes et al. (2003), copyright 2003 American Geophysical Union. Reproduced/modified by permission of American Geophysical Union.
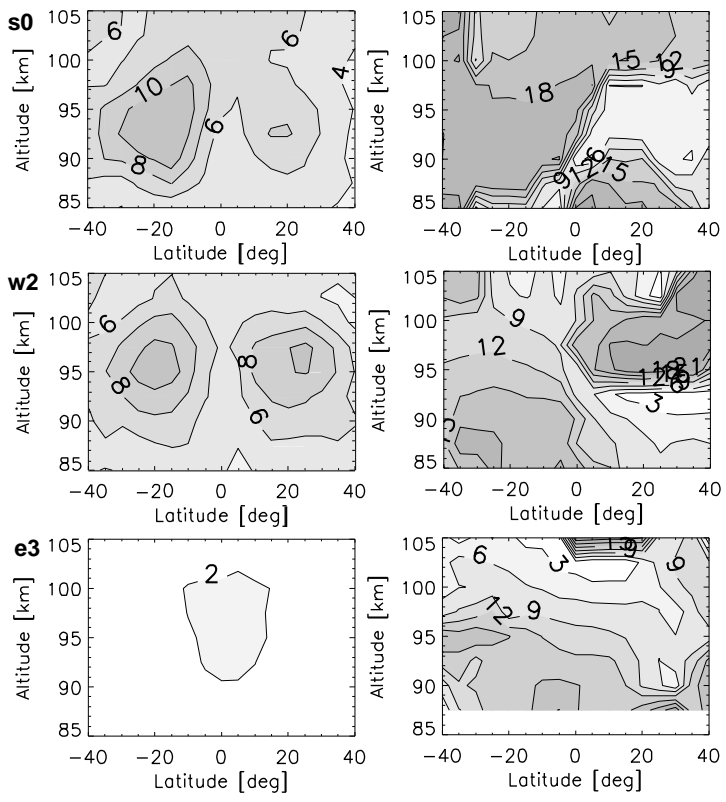

Fig. 2. Left panel: Annual mean diurnal tidal amplitudes for the meridional wind in $\mathrm{m} / \mathrm{s}$. Right panel: Corresponding phases (UT of maximum at $0^{\circ}$ longitude in hours) for a representative month. Top to bottom: $s 0$ (September), $w 2$ (October), $e 3$ (February). Small amplitudes prevent a phase determination below $88 \mathrm{~km}$ altitude for $e 3$. 

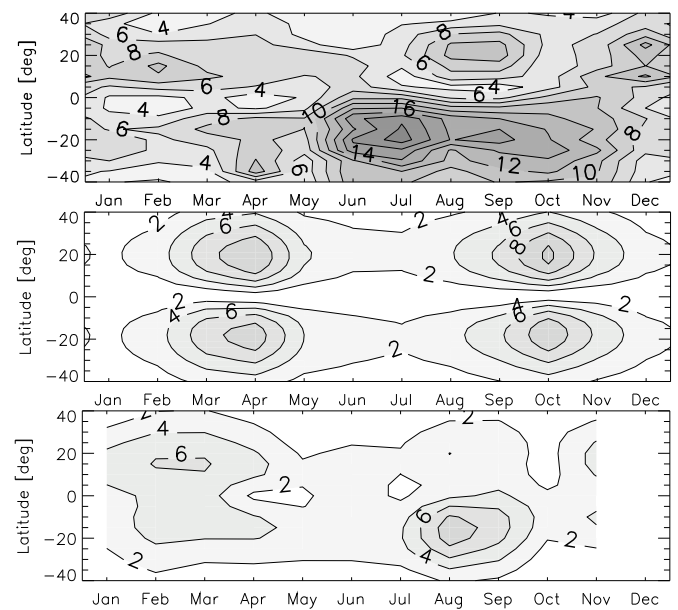

Fig. 3. Comparison between the $95 \mathrm{~km}$ amplitudes $(\mathrm{m} / \mathrm{s})$ of component $s 0$ from TIDI (top), GSWM (middle, latent heat forcing only), and TIME-GCM (bottom). Both models together can explain a large part of the observed amplitudes.

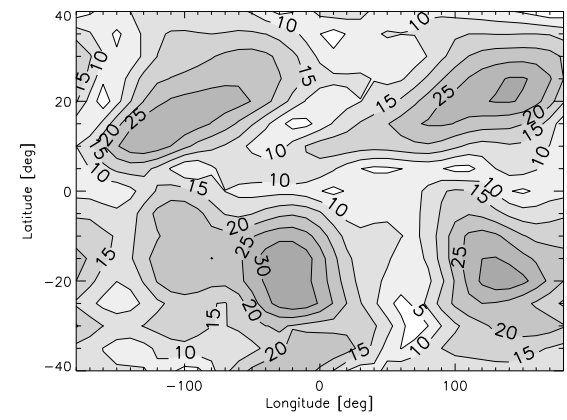

Fig. 4. Longitude/latitude distribution of the nonmigrating diurnal tidal amplitudes (m/s, meridional wind) for October at $95 \mathrm{~km}$. A considerable longitude modulation is observed. 